\title{
Captures of Hot and Warm Sterile Antineutrino Dark Matter on EC-decaying ${ }^{163}$ Ho Nuclei
}

\author{
Y.F. Li ${ }^{\text {a } 1}$ and Zhi-zhong Xing a b 2 \\ ${ }^{a}$ Institute of High Energy Physics, Chinese Academy of Sciences, Beijing 100049, China \\ ${ }^{b}$ Center for High Energy Physics, Peking University, Beijing 100080, China
}

\begin{abstract}
Capturing low-energy electron antineutrinos on radioactive ${ }^{163}$ Ho nuclei, which decay into ${ }^{163}$ Dy via electron capture (EC), is a noteworthy opportunity to detect relic sterile antineutrinos. Such hypothetical particles are more or less implied by current experimental and cosmological data, and they might be a part of hot dark matter or a candidate for warm dark matter in the Universe. Using the isotope ${ }^{163} \mathrm{Ho}$ as a target and assuming reasonable active-sterile antineutrino mixing angles, we calculate the capture rate of relic electron antineutrinos against the corresponding EC-decay background in the presence of sterile antineutrinos at the sub-eV or keV mass scale. We show that the signature of hot or warm sterile antineutrino dark matter should in principle be observable, provided the target is big enough and the energy resolution is good enough.
\end{abstract}

Keywords: sterile antineutrinos; isotope ${ }^{163} \mathrm{Ho}$; hot dark matter; warm dark matter

\footnotetext{
${ }^{1}$ E-mail: liyufeng@ihep.ac.cn

${ }^{2}$ E-mail: xingzz@ihep.ac.cn
} 


\section{Introduction}

Although the existence of dark matter (DM) in the Universe has been established, what it is made of remains a fundamental puzzle [1]. Within the standard model (SM) three kinds of active neutrinos and their antiparticles, whose masses lie in the sub-eV range, may constitute hot DM. Beyond the SM one or more species of sterile neutrinos and antineutrinos at a similar mass scale may also form hot DM, if they were thermalized in the early Universe as their active counterparts. Such light sterile particles are hypothetical, but their existence is more or less implied by current experimental and cosmological data. On the one hand, the long-standing LSND antineutrino anomaly [2], the more recent MiniBooNE antineutrino anomaly [3] and the latest reactor antineutrino anomaly [4] can all be interpreted as the active-sterile antineutrino oscillations in the assumption of two kinds of sterile antineutrinos whose masses are close to $1 \mathrm{eV}[5]$. On the other hand, an analysis of the existing data on the cosmic microwave background (CMB), galaxy clustering and supernovae Ia favors some extra radiation content in the Universe and one or two species of sterile neutrinos and antineutrinos at the sub-eV mass scale [6] ${ }^{1}$. We are therefore open-minded to conjecture that hot DM may in general consist of both active and sterile components. These relics of the Big Bang form the unseen cosmic neutrino background $(\mathrm{C} \nu \mathrm{B})$ and cosmic antineutrino background $(\mathrm{C} \bar{\nu} \mathrm{B})$, whose temperature $T_{\nu}$ is slightly lower than the CMB temperature $T_{\gamma}$ (i.e., $T_{\nu}=\sqrt[3]{4 / 11} T_{\gamma} \simeq 1.945 \mathrm{eV}$ today).

But hot DM only has a tiny contribution to the total matter density of the Universe. A careful analysis of the structure formation indicates that most DM should be cold (nonrelativistic) or warm (semirelativistic) at the onset of the galaxy formation, when the temperature of the Universe was about $1 \mathrm{keV}[8]$. A number of candidates for cold DM, such as weakly interacting massive particles and axions [9], have so far been investigated. In comparison, warm DM is another interesting possibility of accounting for the observed non-luminous and non-baryonic matter content in the Universe. Its existence may allow us to solve or soften several problems that we have recently encountered in the DM simulations [10] (e.g., to damp the inhomogeneities on small scales by reducing the number of dwarf galaxies or to smooth the cusps in the DM halos). A good candidate for warm DM should be sterile neutrinos and antineutrinos, if their masses are in the $\mathrm{keV}$ range and their lifetimes are much longer than the age of the Universe [11]. They could be produced in the early Universe in several ways [12, and should be able to suppress the formation of dwarf galaxies and other small-scale structures and have impacts on the X-ray spectrum, the velocity distribution of pulsars and the formation of the first stars [13]. Hence it is likely to constrain their masses and mixing angles by measuring the Lyman- $\alpha$ forest and $\mathrm{X}$-ray fluxes [14]. In this connection some preliminary hints of keV sterile neutrinos and antineutrinos have recently been discussed [15].

On the theoretical side, there are some interesting models which can accommodate sterile neutrinos and antineutrinos at either keV [16] or sub-eV [17] mass scales. A modelindependent argument [18] is also supporting the conjecture of warm DM hiding out in the "flavor desert" of the SM fermion mass spectrum [19]. Here our main concern is purely phenomenological: how can one directly probe the sterile component of the $\mathrm{C} \bar{\nu} \mathrm{B}$ and the $\mathrm{keV}$ sterile antineutrino DM? In Ref. [20] it has been shown that the sterile component of the $\mathrm{C} \nu \mathrm{B}$ can in principle be detected by means of the thresholdless reaction

\footnotetext{
${ }^{1}$ If the Big Bang nucleosynthesis (BBN) bound is taken into account, however, only one species of light sterile neutrinos and antineutrinos is allowed [7].
} 
$\nu_{e}+{ }^{3} \mathrm{H} \rightarrow{ }^{3} \mathrm{He}+e^{-}$, because the mass eigenstates of sub-eV sterile neutrinos contribute to $\nu_{e}$ and thus leaves a distinct imprint on the electron energy spectrum when they are captured on ${ }^{3} \mathrm{H}$ nuclei 2 . The same idea has also been used to capture the keV sterile neutrino $\mathrm{DM}$ on radioactive $\beta$-decaying ${ }^{3} \mathrm{H}$ and ${ }^{106} \mathrm{Ru}$ nuclei [19, 27]. However, this approach does not directly apply to the capture of light sterile antineutrinos, simply because it is $\nu_{e}$ (instead of $\bar{\nu}_{e}$ ) that is involved in the capture reaction.

A possible way out is to make use of some radioactive nuclei which can decay via electron capture (EC) [28]. It has recently been pointed out that the isotope ${ }^{163} \mathrm{Ho}$, which undergoes an EC decay into ${ }^{163}$ Dy with a small energy release $(Q \simeq 2.5 \mathrm{keV})$, may serve as a target to directly detect the active component of the $\mathrm{C} \bar{\nu} \mathrm{B}$ [29]. This method also works in the presence of flavor effects, which are found to be appreciable and even important in some cases [30]. Here we shall adopt the same idea to probe the sterile component of the $\mathrm{C} \bar{\nu} \mathrm{B}$ and the keV sterile antineutrino DM. The EC decay of ${ }^{163} \mathrm{Ho}$ [31] can be written as

$$
{ }^{163} \mathrm{Ho}+e_{i(\text { shell })}^{-} \rightarrow{ }^{163} \mathrm{Dy}_{i}^{*}+\nu_{e} \rightarrow{ }^{163} \mathrm{Dy}+E_{i}+\nu_{e}
$$

and a thresholdless capture of the incoming $\bar{\nu}_{e}$ on the EC-decaying ${ }^{163}$ Ho may happen via

$$
\bar{\nu}_{e}+{ }^{163} \mathrm{Ho}+e_{i(\text { shell })}^{-} \rightarrow{ }^{163} \mathrm{Dy}_{i}^{*} \rightarrow{ }^{163} \mathrm{Dy}+E_{i}
$$

where $e_{i \text { (shell) }}^{-}$is an orbital electron from the $i$-th shell of ${ }^{163} \mathrm{Ho}$, and $E_{i}$ is the corresponding binding energy of the electron hole in ${ }^{163} \mathrm{Dy}$. Our main purpose is to identify a signal shown in Eq. (2) from the background described by Eq. (1) in the presence of relic sub-eV or keV sterile antineutrinos. Such a study has not been done before and makes sense at least in the following three aspects. First, it is a useful and nontrivial extension of the discussions about the active component of the $\mathrm{C} \bar{\nu} \mathrm{B}$ in Refs. [29] and [30] and may provide us with a novel approach towards the direct detection of relic sterile antineutrinos, which should be compared with relic sterile neutrinos. Second, it illustrates a common way to probe hot and warm sterile antineutrino DM. Although this way is extremely challenging and its prospect is rather remote, it is hitherto the most promising way in this connection. Third, it may serve to highlight the importance of doing a high-statistics calorimetric experiment to measure the ${ }^{163}$ Ho spectrum, probe the absolute neutrino mass scale [32] and even detect the $\mathrm{C} \bar{\nu} \mathrm{B}$ and warm sterile antineutrino DM.

The remaining parts of this paper are organized as follows. In section 2 we summarize the main formulas which can be used to calculate the energy spectrum of the EC decay in Eq. (1) and the rate of the relic antineutrino capture in Eq. (2). Section 3 is devoted to the capture of hot sterile antineutrino DM in the $(3+2)$ scheme of active-sterile antineutrino mixing. We show that the signature is located on the right-hand side of the spectral endpoint of the EC decay, and their interval is detectable if the target is big enough, the energy resolution is good enough and the gravitational clustering of sub-eV sterile antineutrinos is significant around the Earth. Section 4 is devoted to the capture of warm sterile antineutrino DM on ${ }^{163}$ Ho nuclei. We calculate the capture rate by assuming the existence of one species of sterile antineutrinos at the keV mass scale. We find that it is in principle possible to identify a signal of this kind of warm DM, but the required target mass is too big to be accomplishable in the foreseeable future. We conclude in section 5 .

\footnotetext{
${ }^{2}$ This approach was first proposed by Weinberg [21] and by Irvine and Humphreys [22] to detect the active component of the $\mathrm{C} \nu \mathrm{B}$. It has recently attracted more interest because it seems to be the most promising possibility for the direct laboratory detection of relic neutrinos (see Refs. [20] and [23] - 26]).
} 


\section{Relic antineutrino captures}

The key point of relic antineutrino captures on the EC-dcaying ${ }^{163}$ Ho nuclei is to capture the relic electron antineutrinos no matter how low their kinetic energies are. In the presence of $N_{\mathrm{s}}$ species of light sterile neutrinos and antineutrinos, the flavor eigenstates $\left|\nu_{e}\right\rangle$ and $\left|\bar{\nu}_{e}\right\rangle$ can be written as

$$
\begin{aligned}
& \left|\nu_{e}\right\rangle=\sum_{k} V_{e k}^{*}\left|\nu_{k}\right\rangle, \\
& \left|\bar{\nu}_{e}\right\rangle=\sum_{k} V_{e k}\left|\bar{\nu}_{k}\right\rangle,
\end{aligned}
$$

where $\left|\nu_{k}\right\rangle$ (or $\left|\bar{\nu}_{k}\right\rangle$ ) stands for the mass eigenstate of an active (for $1 \leq k \leq 3$ ) or sterile (for $4 \leq k \leq N_{\mathrm{s}}$ ) neutrino (or antineutrino), and $V_{e k}$ denotes an element in the first row of the $\left(3+N_{\mathrm{s}}\right) \times\left(3+N_{\mathrm{s}}\right)$ neutrino mixing matrix $V$ [33]. Given current experimental constraints on sterile antineutrinos [5], it is reasonable to assume that the light sterile antineutrinos under consideration do not significantly affect the values of two mass-squared differences and three mixing angles of three active antineutrinos extracted from solar, atmospheric, reactor and accelerator neutrino oscillation data [8]. In this assumption we shall use $\Delta m_{21}^{2} \simeq 7.6 \times 10^{-5} \mathrm{eV}^{2}$ and $\left|\Delta m_{31}^{2}\right| \simeq 2.4 \times 10^{-3} \mathrm{eV}^{2}$ together with $\theta_{12} \simeq 34^{\circ}$ and $\theta_{13} \simeq 10^{\circ}$ as typical inputs for our analysis. Depending on the sign of $\Delta m_{31}^{2}$, there are two possible mass patterns for active antineutrinos: $m_{1}<m_{2}<m_{3}$ (normal hierarchy) or $m_{3}<m_{1}<m_{2}$ (inverted hierarchy). In either case the absolute mass scale is unknown, but its upper bound is expected to be of $\mathcal{O}(0.1) \mathrm{eV}$ as constrained by current cosmological data [34]. We shall specify the values of $m_{k}$ and $\left|V_{e k}\right|$ (for $\left.k=4, \cdots, N_{\mathrm{s}}\right)$ when we numerically calculate the capture rates of relic sterile antineutrinos in sections 3 and 4 .

Now let us consider the EC decay of ${ }^{163} \mathrm{Ho}$ in Eq. (1). If the $Q$-value of this reaction is defined as the mass difference between ${ }^{163} \mathrm{Ho}$ and ${ }^{163} \mathrm{Dy}$, then the energy spectrum of the outgoing neutrinos will be given by a series of lines at $Q-E_{i}$. For the time being $Q$ has been constrained to the range $2.3 \mathrm{keV} \leq Q \leq 2.8 \mathrm{keV}$ [29], and we shall typically take $Q \simeq 2.5 \mathrm{keV}$ in our numerical calculations. Taking account of the BreitWigner resonance form of the atomic levels, one has obtained the energy spectrum of the detected EC events [31] and its integral in the narrow-width approximation [35]. As for the thresholdless capture of the incoming relic antineutrinos on the EC-decaying ${ }^{163} \mathrm{Ho}$ nuclei in Eq. (2), the de-excitation energy of unstable ${ }^{163} \mathrm{Dy}_{i}^{*}$ is in principle monoenergetic for each antineutrino mass eigenstate $\bar{\nu}_{k}$ (i.e., $T_{k} \equiv E_{\bar{\nu}_{k}}+Q$ ). Convoluted with a finite energy resolution in a realistic experiment, the ideally discrete energy lines of the final states in Eq. (2) must spread and then form a continuous spectrum. As usual, we adopt a Gaussian energy resolution function defined by

$$
R\left(T, T_{k}\right)=\frac{1}{\sqrt{2 \pi} \sigma} \exp \left[-\frac{\left(T-T_{k}\right)^{2}}{2 \sigma^{2}}\right],
$$

where $T$ is the overall energy of an event detected in the experiment. Using $\Delta$ to denote the experimental energy resolution (i.e., the full width at half maximum of a Gaussian energy resolution for the detected events), we have $\Delta=2 \sqrt{2 \ln 2} \sigma \simeq 2.35482 \sigma$. Then the differential antineutrino capture rate reads [30]

$$
\frac{\mathrm{d} \lambda_{\bar{\nu}}}{\mathrm{d} T}=\frac{G_{\beta}^{2}}{2} \sum_{i} \sum_{k} n_{\bar{\nu}_{k}}\left|V_{e k}\right|^{2} R\left(T, T_{k}\right) n_{i} C_{i} \beta_{i}^{2} B_{i} \frac{\Gamma_{i}}{2 \pi} \cdot \frac{1}{\left(T_{k}-E_{i}\right)^{2}+\Gamma_{i}^{2} / 4},
$$


where $G_{\beta} \equiv G_{\mathrm{F}} \cos \theta_{\mathrm{C}}$ with $\theta_{\mathrm{C}} \simeq 13^{\circ}$ being the Cabibbo angle of quark flavor mixing, $n_{\bar{\nu}_{k}}$ denotes the number density of $\bar{\nu}_{k}, n_{i}$ is the fraction of occupancy of the $i$-th electron shell, $C_{i}$ stands for the nuclear shape factor, $\beta_{i}$ represents the Coulomb amplitude of the electron radial wave function, $B_{i}$ is an atomic correction for the electron exchange and overlap, and $\Gamma_{i}$ denotes the finite natural width of the $i$-th atomic level. The $Q$-value of the EC decay in Eq. (1) is so small that only those electrons from $M_{1}, M_{2}, N_{1}, N_{2}, O_{1}$, $\mathrm{O}_{2}$ and $P_{1}$ levels can be captured [29]. In accordance with Eq. (5), the energy spectrum of the EC decay should also be convoluted with the Gaussian energy resolution [30]:

$$
\begin{aligned}
\frac{\mathrm{d} \lambda_{\mathrm{EC}}}{\mathrm{d} T}= & \int_{0}^{Q-\min \left(m_{k}\right)} \mathrm{d} T_{\mathrm{c}}\left[\frac{G_{\beta}^{2}}{4 \pi^{2}} R\left(T, T_{\mathrm{c}}\right)\left(Q-T_{\mathrm{c}}\right)\right. \\
& \times \sum_{k}\left|V_{e k}\right|^{2} \sqrt{\left(Q-T_{\mathrm{c}}\right)^{2}-m_{k}^{2}} \Theta\left(Q-T_{\mathrm{c}}-m_{k}\right) \\
& \left.\times \sum_{i} n_{i} C_{i} \beta_{i}^{2} B_{i} \frac{\Gamma_{i}}{2 \pi} \cdot \frac{1}{\left(T_{\mathrm{c}}-E_{i}\right)^{2}+\Gamma_{i}^{2} / 4}\right]
\end{aligned}
$$

where the theta function $\Theta\left(Q-T_{\mathrm{c}}-m_{k}\right)$ has been introduced to ensure the kinematic requirement. One may use Eqs. (5) and (6) to calculate the rate of the relic antineutrino capture on the EC-decaying ${ }^{163}$ Ho nuclei against the corresponding background (i.e., the EC decay itself) in the presence of active and sterile flavor effects, which are characterized by both the antineutrino masses $m_{k}$ and the antineutrino mixing matrix elements $\left|V_{e k}\right|$ (for $\left.k=1,2, \cdots N_{\mathrm{s}}\right)$. It is therefore possible to probe the existence of relic sterile antineutrinos at either sub-eV or keV mass scales.

In our numerical calculations we shall input the values of those parameters relevant to the atomic levels of ${ }^{163}$ Dy given in Ref. [29] and references therein. The energy levels of the captured electrons are assumed to be fully occupied (i.e., $n_{i} \simeq 1$ ) and their binding energies and widths can be found in Table 1 of Ref. [29]. The atomic corrections for the electron exchange and overlap are neglected (i.e., $B_{i} \simeq 1$ ), and the ratios of the squared wave functions at the origin (i.e., $\beta_{i}^{2} / \beta_{\mathrm{M}_{1}}^{2}$ ) can be found in Table 2 of Ref. [29]. Because the nuclear shape factors $C_{i}$ are approximately identical in an allowed transition [31], they can be factored out from the sum in Eqs. (5) and (6). Note that the numerical results of $\lambda_{\bar{\nu}}$ and $\lambda_{\mathrm{EC}}$ can be properly normalized by using the half-life of ${ }^{163} \mathrm{Ho}$ via the relation

$\lambda_{\mathrm{EC}} T_{1 / 2}=\ln 2$, where $T_{1 / 2} \simeq 4570 \mathrm{yr}$. Then the distributions of the number of signal events and the number of background events are expressed, respectively, as

$$
\begin{aligned}
& \frac{\mathrm{d} N_{\mathrm{S}}}{\mathrm{d} T}=\frac{1}{\lambda_{\mathrm{EC}}} \cdot \frac{\mathrm{d} \lambda_{\bar{\nu}}}{\mathrm{d} T} \cdot \frac{\ln 2}{T_{1 / 2}} N_{\mathrm{T}} t, \\
& \frac{\mathrm{d} N_{\mathrm{B}}}{\mathrm{d} T}=\frac{1}{\lambda_{\mathrm{EC}}} \cdot \frac{\mathrm{d} \lambda_{\mathrm{EC}}}{\mathrm{d} T} \cdot \frac{\ln 2}{T_{1 / 2}} N_{\mathrm{T}} t
\end{aligned}
$$

for a given target factor $N_{\mathrm{T}}$ (i.e., the number of ${ }^{163} \mathrm{Ho}$ atoms of the target) and for a given exposure time $t$ in the experiment.

\section{Hot sterile antineutrino DM}

To be explicit, let us focus on the capture of hot sterile antineutrino DM in the $(3+2)$ flavor mixing scheme with two species of sub-eV sterile neutrinos and antineutrinos [5]. 
The absolute mass scale of three active antineutrinos is characterized by $m_{1}$ (normal hierarchy) or $m_{3}$ (inverted hierarchy), and its value is typically taken to be $0.0 \mathrm{eV}, 0.05$ $\mathrm{eV}$ or $0.1 \mathrm{eV}$ in our numerical analysis. In addition, we choose $m_{1} \simeq 0.2 \mathrm{eV}$ and $m_{5} \simeq 0.4$ $\mathrm{eV}$ together with $\theta_{14} \simeq \theta_{15} \approx 10^{\circ}$ for two sterile antineutrinos 3 . Accordingly, we have $\left|V_{e 1}\right| \simeq 0.792,\left|V_{e 2}\right| \simeq 0.534,\left|V_{e 3}\right| \simeq 0.168,\left|V_{e 4}\right| \simeq 0.171$ and $\left|V_{e 5}\right| \simeq 0.174$. Such sterile antineutrinos are expected to be cosmologically friendly [6], and they should stay in full thermal equilibrium in the early Universe [36]. In this case the standard Big Bang model predicts the average number density $\left\langle n_{\nu_{k}}\right\rangle \simeq\left\langle n_{\bar{\nu}_{k}}\right\rangle \simeq 56 \mathrm{~cm}^{-3}$ today for each species of active and sterile neutrinos and antineutrinos.

For our purpose, however, we are mainly concerned about the number density of relic antineutrinos around the Earth because its value $n_{\bar{\nu}_{k}}$ may be more or less enhanced by the gravitational clustering effect. A detailed analysis of gravitational clustering of relic neutrinos and antineutrinos in our local neighborhood has been done in Ref. [37]. Here we take account of this effect by conjecturing a simplified power law relation between the relic antineutrino overdensity parameter $\zeta_{k} \equiv n_{\bar{\nu}_{k}} /\left\langle n_{\bar{\nu}_{k}}\right\rangle$ and the corresponding antineutrino mass $m_{k}\left(\right.$ for $\left.k=1,2, \cdots, N_{\mathrm{s}}\right)$ :

$$
\zeta_{k} \simeq 1+A\left(\frac{m_{k}}{1 \mathrm{eV}}\right)^{\Omega}
$$

where $A$ and $\Omega$ are independent of the subscript $k$. Note that the size of $\zeta_{k}$ for a given value of $m_{k}$ should lie between the results obtained in two extreme cases as summarized in Table 2 of Ref. [37]. For simplicity, here we take the average of every pair of those extreme values as the central input of $\zeta_{k}$ and one half of their difference as the error bar of $\zeta_{k}$. Then we have $\zeta_{k}=\{1.5 \pm 0.1,3.75 \pm 0.65,8.2 \pm 1.8,16 \pm 4\}$ corresponding to $m_{k}=\{0.15,0.3,0.45,0.6\} \mathrm{eV}$ extracted from Table 2 in Ref. [37. After a least square analysis of the relation between $m_{k}$ and $\zeta_{k}$ as illustrated in Fig. 1, we find that the best-fit values of $A$ and $\Omega$ are $\log _{10} A \simeq 1.7$ and $\Omega \simeq 2.4$. One may easily see that the deviation of $\zeta_{k}$ from one becomes significant only when $m_{k}$ is larger than $0.1 \mathrm{eV}$ (e.g., $\zeta_{k} \simeq 2$ for $\left.m_{k} \simeq 0.2 \mathrm{eV}\right)$. So in the $(3+2)$ flavor mixing scheme under discussion, only two sub-eV sterile antineutrinos are relatively sensitive to the gravitational clustering effect.

We calculate the rate of the relic antineutrino capture on ${ }^{163} \mathrm{Ho}$ nuclei against the rate of the corresponding EC decay by means of Eqs. (5), (6) and (7). Our numerical results are presented in Figs. 2 and 3. Some discussions are in order.

(1) Fig. 2 illustrates the relic antineutrino capture rate as a function of the overall energy release $T$ in the case of $\Delta m_{31}^{2}>0$. In the left panel $\zeta_{k}=1$ (i.e., $n_{\bar{\nu}_{k}}=\left\langle n_{\bar{\nu}_{k}}\right\rangle$ ) is assumed, and in the right panel the gravitational clustering effect (i.e., $\zeta_{k}>1$ ) has roughly been taken into account with the help of Eq. (8). The impact of different values of $m_{1}$ on the capture rate can be vertically seen in each panel. Note that the value of the finite energy resolution $\Delta$ is taken in such a way that only the signal of hot sterile antineutrino DM can be observed, since the signal of hot active antineutrino DM has already been discussed in Ref. [30]. We find that it is in principle possible to distinguish the signal from the background when $\Delta$ is smaller than $0.1 \mathrm{eV}$. As the value of $m_{1}$ increases from 0

\footnotetext{
${ }^{3}$ The values of two sterile antineutrino masses taken here are more favored by current cosmological data 6, but they are somewhat smaller than those values extracted from a global fit of the LSND, MiniBooNE and reactor antineutrino anomalies [5]. It is simply a matter of taste for us to take the cosmological hints on sub-eV sterile neutrinos and antineutrinos more seriously. Since the chosen values of $m_{4}$ and $m_{5}$ are mainly for the purpose of illustration, they do not qualitatively affect our conclusions.
} 
to $0.1 \mathrm{eV}$, the signal curve moves towards the higher $T-Q$ region while the background curve moves towards the lower $T-Q$ region. Hence the interval between the peak of a signal and the background becomes larger for a larger value of $m_{1}$, making it easier to detect this signal. If the heights of two neighboring signals are quite different and their locations satisfy $m_{i}-m_{j} \sim \Delta$, it will be difficult to distinguish between them because the higher signal is much broader and may essentially overwhelm the lower one. This feature can be seen in Fig. 2(e) and Fig. 2(f), where the signal of $\bar{\nu}_{4}$ and the signal of three active antineutrinos with nearly degenerate masses merge into a single one. In this case only the signal of much heavier $\bar{\nu}_{5}$ is clearly distinguishable. In the right panel of Fig. 2 one may observe that the gravitational clustering effect can enhance the capture rate, and this effect is more significant for heavier sterile antineutrinos. If the gravitational clustering of relic antineutrinos were much more significant around the Earth, it would be very helpful for us to detect the $\mathrm{C} \bar{\nu} \mathrm{B}$ by means of the capture reaction under consideration.

(2) Fig. 3 shows the numerical results obtained from an analogous analysis of the relic antineutrino capture rate in the case of $\Delta m_{31}^{2}<0$. We see that it is quite similar to Fig. 2, and their main differences appear in the location of the signal of three active antineutrinos and that of the endpoint of the EC-decay background. The first difference arises from the fact that the location of the signal of active antineutrinos is dominated by the mass eigenstates $\bar{\nu}_{1}$ and $\bar{\nu}_{2}$ which have slightly larger eigenvalues in the $\Delta m_{31}^{2}<0$ case than in the $\Delta m_{31}^{2}>0$ case. As for the second difference, the spectral endpoint of the EC decay of ${ }^{163} \mathrm{Ho}$ is located at a smaller $T-Q$ region in the $\Delta m_{31}^{2}<0$ case, simply because the lightest mass eigenstate $\bar{\nu}_{3}$ hiding out in $\bar{\nu}_{e}$ is associated with the smallest active antineutrino mixing matrix element $\left|V_{e 3}\right|$. So we conclude that it is somewhat easier to observe the signals of hot active and sterile antineutrino DM when three active antineutrinos have an inverted mass hierarchy.

(3) Besides the energy resolution, another factor that obviously affects the observability of hot antineutrino DM is the absolute capture rate which depends on the number density of relic antineutrinos $n_{\bar{\nu}_{k}}$, the flavor mixing matrix elements $\left|V_{e k}\right|$ and the number of the target particles $N_{\mathrm{T}}$. By using the default values of $\left|V_{e k}\right|$ taken above, we illustrate the iso-rate curves for the sterile antineutrino overdensity parameter $\zeta_{5}$ versus the target mass in Fig. 44 . Because of $\left|V_{e 4}\right| \sim\left|V_{e 5}\right|$ and $m_{4} \sim m_{5} \ll Q-E_{i}$, one may obtain very similar iso-rate curves for $\zeta_{4}$ versus the target mass. These curves are simply straight lines in the double logarithmic scale, which can easily be understood with the help of Eqs. (5) and (7). Given $\zeta_{k}=10$, for example, a target with $10^{2} \mathrm{~kg}$ (or $10^{3} \mathrm{~kg}$ ) ${ }^{163} \mathrm{Ho}$ could be enough to give a capture rate of one event per year (or ten events per year).

Finally, let us give a brief comment on the properties of the capture signals of hot sterile antineutrinos against the corresponding EC decays by taking the masses of sterile antineutrinos to be consistent with the values of $\Delta m_{41}^{2}$ and $\Delta m_{51}^{2}$ extracted from a global fit of the LSND, MiniBooNE and reactor antineutrino anomalies (i.e., $\Delta m_{41}^{2} \simeq 0.47 \mathrm{eV}^{2}$ and $\left.\Delta m_{51}^{2} \simeq 0.87 \mathrm{eV}^{2}[5]\right)$. In this case the dependence of the capture rates on the energy resolution, antineutrino number densities and target factors is quite similar to the one discussed above. The only change is the location of each sterile antineutrino signal. Because of $m_{4} \simeq \sqrt{\Delta m_{41}^{2}} \simeq 0.69 \mathrm{eV}$ and $m_{5} \simeq \sqrt{\Delta m_{51}^{2}} \simeq 0.93 \mathrm{eV}$ in the present situation,

\footnotetext{
${ }^{4}$ Instead of using Eq. (8), here we treat $\zeta_{5}$ as a free parameter independent of the neutrino masses $m_{k}$. In the literature there are some different neutrino overdensity models which can actually predict very large values of $\zeta_{k}$ (see, e.g., Ref. [38).
} 
it will be much easier to identify the sterile antineutrino signals on EC-decaying ${ }^{163} \mathrm{Ho}$ nuclei if the magnitudes of the mixing matrix elements $\left|V_{e 4}\right|$ and $\left|V_{e 5}\right|$ keep unchanged.

\section{Warm sterile antineutrino DM}

Now we turn to the capture of warm sterile antineutrino DM in the $(3+1)$ flavor mixing scheme with one species of $\mathrm{keV}$ sterile neutrinos and antineutrinos. To be explicit, we fix $m_{4} \simeq 2.0 \mathrm{keV}$ and $\left|V_{e 4}\right|^{2} \simeq 5.0 \times 10^{-7}$ in our numerical analysis, just for the purpose of illustration [19]. Because our main concern is to observe a possible signal of the relic antineutrino capture in the keV mass region, we simply assume three active antineutrinos to have a normal mass hierarchy with $m_{1}=0$. A very similar signal can also be obtained for the inverted or nearly degenerate mass pattern of three active antineutrinos. One may also consider the gravitational clustering effect on the $\mathrm{C} \bar{\nu} \mathrm{B}$ as described in Eq. (8), but it is insignificant in the present scenario where only active antineutrinos contribute to the $\mathrm{C} \bar{\nu} \mathrm{B}$. As for the $\mathrm{keV}$ sterile neutrinos and antineutrinos, we follow Ref. [12] to assume that they were produced in the early Universe through active-sterile neutrino or antineutrino oscillations and their number densities are able to account for the total amount of DM. Given the average density of DM in our Galactic neighborhood (i.e., $\left.\rho_{\mathrm{DM}}^{\text {local }} \simeq 0.3 \mathrm{GeV} \mathrm{cm}^{-3}[39]\right)$, it is straightforward to estimate the number density of $\nu_{4}$ or $\bar{\nu}_{4}$. We obtain $n_{\nu_{4}} \simeq n_{\bar{\nu}_{4}} \simeq 5 \times 10^{4}\left(3 \mathrm{keV} / m_{4}\right) \mathrm{cm}^{-3}$.

We calculate the capture rate of warm sterile antineutrino DM on ${ }^{163} \mathrm{Ho}$ nuclei against the corresponding EC-decay background by using Eqs. (5), (6) and (7). Our numerical result is shown in Fig. 5. Some discussions are in order.

(1) In obtaining Fig. 5 we have chosen a typical value of the finite energy resolution $\Delta$ to distinguish the signal from the background. The endpoint of the background is sensitive to $\Delta$, while the peak of the signal is always located at $T=Q+m_{4}$. So a comparison between $\Delta$ and $m_{4}$ can easily reveal the signal-to-background ratio. The required energy resolution to identify the signal of warm sterile antineutrino DM is of $\mathcal{O}(0.1) \mathrm{keV}$, which should be easily reachable in a realistic experiment.

(2) The main problem which makes the observability of keV sterile antineutrino DM rather dim and remote is the tiny capture rate. The latter is strongly suppressed for two simple reasons. On the one hand, the mixing factor between three active antineutrinos and the keV sterile antineutrino is too small [11]. On the other hand, the Breit-Wigner distribution function is too small when the signal of $\bar{\nu}_{4}$ is located far away from the resonance energies $E_{i}$. In Fig. 6 we depict the iso-rate curves for the mixing matrix element $\left|V_{e 4}\right|^{2}$ versus the target mass. One can see that a target with 600 ton ${ }^{163} \mathrm{Ho}$ is needed for $\left|V_{e 4}\right|^{2} \sim \mathcal{O}\left(10^{-6}\right)$, so as to obtain a capture rate of one event per year. Hence it is almost hopeless to capture warm sterile antineutrino DM on the EC-decaying ${ }^{163} \mathrm{Ho}$ nuclei in the foreseeable future. In comparison, it seems somewhat more hopeful to detect warm DM in the form of keV sterile neutrinos on radioactive beta-decaying ${ }^{3} \mathrm{H}$ and ${ }^{106} \mathrm{Ru}$ nuclei in the long term [19].

Finally, let us give some brief comments on the detection prospects for three kinds of relic antineutrinos (i.e., hot active antineutrino DM, hot sterile antineutrino DM and warm sterile antineutrino DM). Given a target made of the isotope ${ }^{163} \mathrm{Ho}$, the capture of each kind of DM in a realistic experiment crucially relies on its energy resolution and target mass. Because three active antineutrinos have relatively large mixing angles and relatively 
small masses, the corresponding hot active antineutrino DM has the largest capture rate but requires the most stringent energy resolution $(\Delta \simeq 0.015 \mathrm{eV}$ or smaller, as discussed in Ref. [30]) for us to distinguish a signal from its background. In contrast, the situation for capturing warm sterile antineutrino DM is just the opposite. Here $\Delta \sim \mathcal{O}(0.1) \mathrm{keV}$ is good enough because of $m_{4} \sim \mathcal{O}(1) \mathrm{keV}$, and this energy resolution requirement can be satisfied even today. But the capture rate of $\bar{\nu}_{4}$ on the EC-decaying ${ }^{163}$ Ho nuclei is so small that the desired target mass has to be formidably large. As we have seen in the above analysis, the capture of hot sterile antineutrino DM at the sub-eV (or eV) mass scale requires a relatively mild energy resolution and a reasonably large target mass as compared with the situations for detecting hot active antineutrino DM and warm sterile antineutrino DM in the same way. So it might be possible to probe the sterile component of the $\mathrm{C} \bar{\nu} \mathrm{B}$ by using the isotope ${ }^{163} \mathrm{Ho}$ as a target in the future.

\section{Concluding remarks}

To pin down what DM is made of has been one of the most important and most challenging problems in particle physics and cosmology. In this paper we have addressed ourselves to the direct laboratory detection of possible contributions of light sterile antineutrinos to DM. Such hypothetical particles might be a part of hot DM if their masses are in the sub$\mathrm{eV}$ (or eV) range, or a good candidate for warm DM if their masses lie in the keV range. Choosing the isotope ${ }^{163} \mathrm{Ho}$ as a target and assuming reasonable active-sterile antineutrino mixing angles, we have calculated the capture rate of relic electron antineutrinos against the corresponding EC-decay background in the presence of sterile antineutrinos at either sub-eV or keV mass scales. Our analysis shows that the signature of hot or warm sterile antineutrino DM should in principle be observable, provided the target is big enough, the energy resolution is good enough and the gravitational clustering effect is significant enough. We admit that our numerical results are quite preliminary and mainly serve for illustration, but we stress that such a direct laboratory search for hot and warm sterile antineutrino DM is fundamentally important and deserves further attention and more detailed investigations.

Why has one chosen the EC-decaying ${ }^{163}$ Ho nuclei as a proper target for probing the antineutrino content of DM? The reason is simply that the captures of relic neutrinos and antineutrinos require different types of radioactive nuclei: $\nu_{e}$ is involved in the capture reactions on the beta-decaying ${ }^{3} \mathrm{H}$ and ${ }^{106} \mathrm{Ru}$ nuclei, while $\bar{\nu}_{e}$ is associated with the capture reactions on the EC-decaying ${ }^{163}$ Ho nuclei. So it makes sense to look for another rather stable isotope which can undergo an EC decay with a much lower $Q$-value, in order to do a more promising experiment for the direct detection of hot and warm antineutrino DM. Although the present experimental techniques are unable to lead us to a guaranteed measurement of relic neutrinos and antineutrinos in the near future, we might have a chance to make a success of this great exploration in the long term.

This work was supported in part by the China Postdoctoral Science Foundation under grant No. 20100480025 (Y.F.L.) and in part by the National Natural Science Foundation of China under grant No. 10875131 (Z.Z.X.). 


\section{References}

[1] See, e.g., Z.Z. Xing and S. Zhou, Neutrinos in Particle Physics, Astronomy and Cosmology (Zhejiang University Press and Springer-Verlag, 2011).

[2] A. Aguilar et al. (LSND Collaboration), Phys. Rev. D 64, 112007 (2001).

[3] A.A. Aguilar-Arevalo et al. (MiniBooNE Collaboration), Phys. Rev. Lett. 105, 181801 (2010).

[4] G. Mention et al., Phys. Rev. D 83, 073006 (2011).

[5] J. Kopp, M. Maltoni, and T. Schwetz, arXiv:1103.4570.

[6] J. Hamann et al., Phys. Rev. Lett. 105, 181301 (2010); E. Giusarma et al., Phys. Rev. D 83, 115023 (2011).

[7] See, e.g., G. Mangano and P.D. Serpico, Phys. Lett. B 701, 296 (2011); and references therein.

[8] Particle Data Group, K. Nakamura et al., J. Phys. G 37, 075021 (2010).

[9] For a recent review with extensive references, see: J.L. Feng, Ann. Rev. Astron. Astrophys. 48, 495 (2010).

[10] P. Bode, J.P. Ostriker, and N. Turok, Astrophys. J. 556, 93 (2001).

[11] For recent reviews with extensive references, see: A. Kusenko, Phys. Rept. 481, 1 (2009); A. Boyarsky, O. Ruchayskiy, and M. Shaposhnikov, Ann. Rev. Nucl. Part. Sci. 59, 191 (2009).

[12] See, e.g., S. Dodelson and L.M. Widrow, Phys. Rev. Lett. 72, 17 (1994); X.D. Shi and G.M. Fuller, Phys. Rev. Lett. 82, 2832 (1999). For recent reviews in this connection, see Ref. [11].

[13] See, e.g., A. Kusenko and G. Segre, Phys. Lett. B 396, 197 (1997); G.M. Fuller et al., Phys. Rev. D 68, 103002 (2003).

[14] See, e.g., K. Abazajian and S.M. Koushiappas, Phys. Rev. D 74, 023527 (2006); A. Boyarsky et al., MNRAS 387, 1361 (2008); A. Boyarsky et al., Phys. Rev. Lett. 102, 201304 (2009); M. Loewenstein, A. Kusenko, and P.L. Biemann, Astrophys. J. 700, 426 (2009).

[15] See, e.g., M. Loewenstein and A. Kusenko, Astrophys. J. 714, 652 (2010); A. Boyarsky et al., MNRAS 407, 1188 (2010); D.A. Prokhorov and J. Silk, arXiv:1001.0215; M.H. Chan and M.C. Chu, arXiv:1009.5872.

[16] See, e.g., T. Asaka, S. Blanchet, and M. Shaposhnikov, Phys. Lett. B 631, 151 (2005); A. Kusenko, Phys. Rev. Lett. 97, 241301 (2006); K. Petraki and A. Kusenko, Phys. Rev. D 77, 065014 (2008); A. Kusenko, F. Takahashi, and T. Yanagida, Phys. Lett. B 693, 144 (2010); M. Lindner, A. Merle, and V. Niro, JCAP 1101, 034 (2011). 
[17] See, e.g., V. Barger, P.F. Perez, and S. Spinner, Phys. Lett. B 696, 509 (2011); and references therein.

[18] Z.Z. Xing, invited plenary talk given at COSMO/CosPA 2010, September 2010, Tokyo.

[19] Y.F. Li and Z.Z. Xing, Phys. Lett. B 695, 205 (2011).

[20] Y.F. Li, Z.Z. Xing, and S. Luo, Phys. Lett. B 692, 261 (2010).

[21] S. Weinberg, Phys. Rev. 128, 1457 (1962).

[22] J.M. Irvine and R. Humphreys, J. Phys. G 9, 847 (1983).

[23] A. Cocco, G. Mangano, and M. Messina, JCAP 0706, 015 (2007); R. Lazauskas, P. Vogel, and C. Volpe, J. Phys. G 35, 025001 (2008).

[24] M. Blennow, Phys. Rev. D 77, 113014 (2008).

[25] For a brief review, see: A. Ringwald, Nucl. Phys. A 827, 501c (2009).

[26] R. Hodak, S. Kovalenko, and F. Simkovic, AIP Conf. Proc. 1180, 50 (2009); A. Kaboth, J.A. Formaggio, and B. Monreal, Phys. Rev. D 82, 062001 (2010); A. Faessler et al., arXiv:1102.1799.

[27] W. Liao, Phys. Rev. D 82, 073001 (2010).

[28] A.G. Cocco, G. Mangano, and M. Messina, Phys. Rev. D 79, 053009 (2009).

[29] M. Lusignoli and M. Vignati, Phys. Lett. B 697, 11 (2011).

[30] Y.F. Li and Z.Z. Xing, Phys. Lett. B 698, 430 (2011); arXiv:1102.2686.

[31] W. Bambynek et al., Rev. Mod. Phys. 49, 77 (1977) [Erratum-ibid. 49, 961 (1977)].

[32] See, e.g., F. Gatti et al., Phys. Lett. B 398, 415 (1997); F. Gatti et al., J. Low Temp. Phys. 151, 603 (2008); A. Nucciotti (MARE Collaboration), arXiv:1012.2290.

[33] S. Goswami and W. Rodejohann, JHEP 0710, 073 (2007).

[34] E. Komatsu et al. (WMAP Collaboration), Astrophys. J. Supp. 192, 18 (2011).

[35] A. De Rujula and M. Lusignoli, Phys. Lett. B 118, 429 (1982).

[36] S. Hannestad and G.G. Raffelt, Phys. Rev. D 59, 043001 (1999).

[37] A. Ringwald and Y.Y.Y. Wong, JCAP 0412, 005 (2004).

[38] R. Wigmans, Astropart. Phys. 19, 379 (2003); W. Hwang and B. Ma, New J. Phys. 7, 41 (2005).

[39] M. Kamionkowski and A. Kinkhabwala, Phys. Rev. D 57, 3256 (1998). 


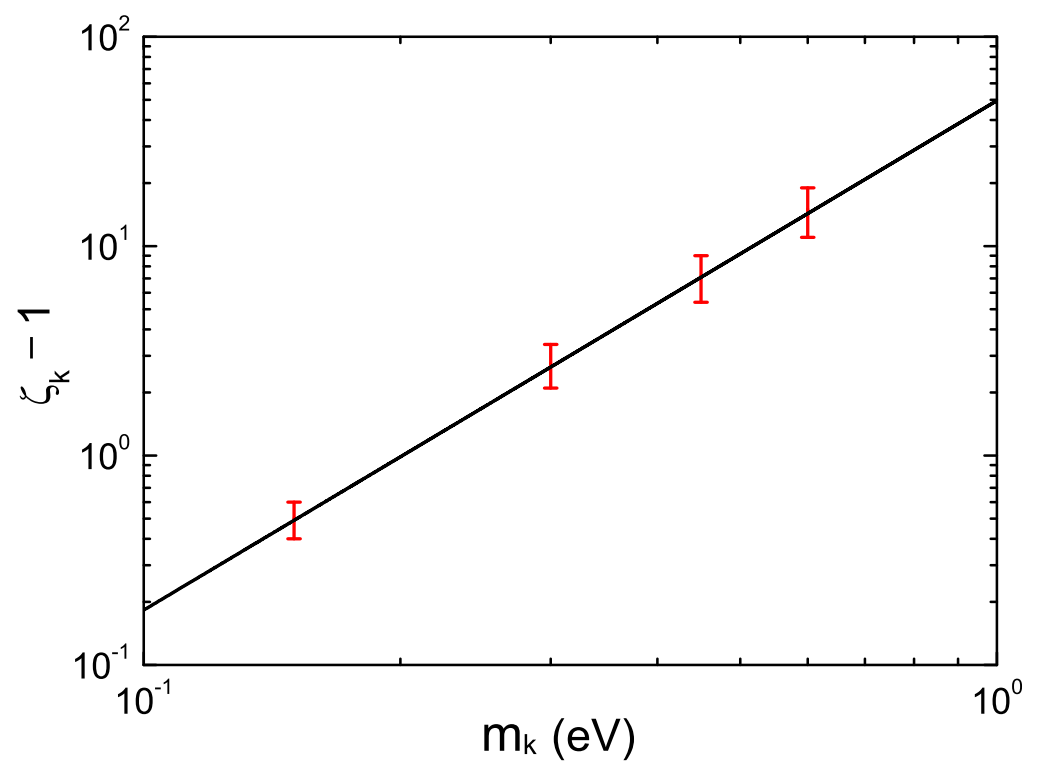

Figure 1: The best-fit power law relation between the antineutrino mass $m_{k}$ and the overdensity parameter $\zeta_{k}$ as a consequence of the gravitational clustering effect on relic antineutrinos in our local neighborhood. The four points and their error bars in this graph are extracted from Table 2 of Ref. [37]. 

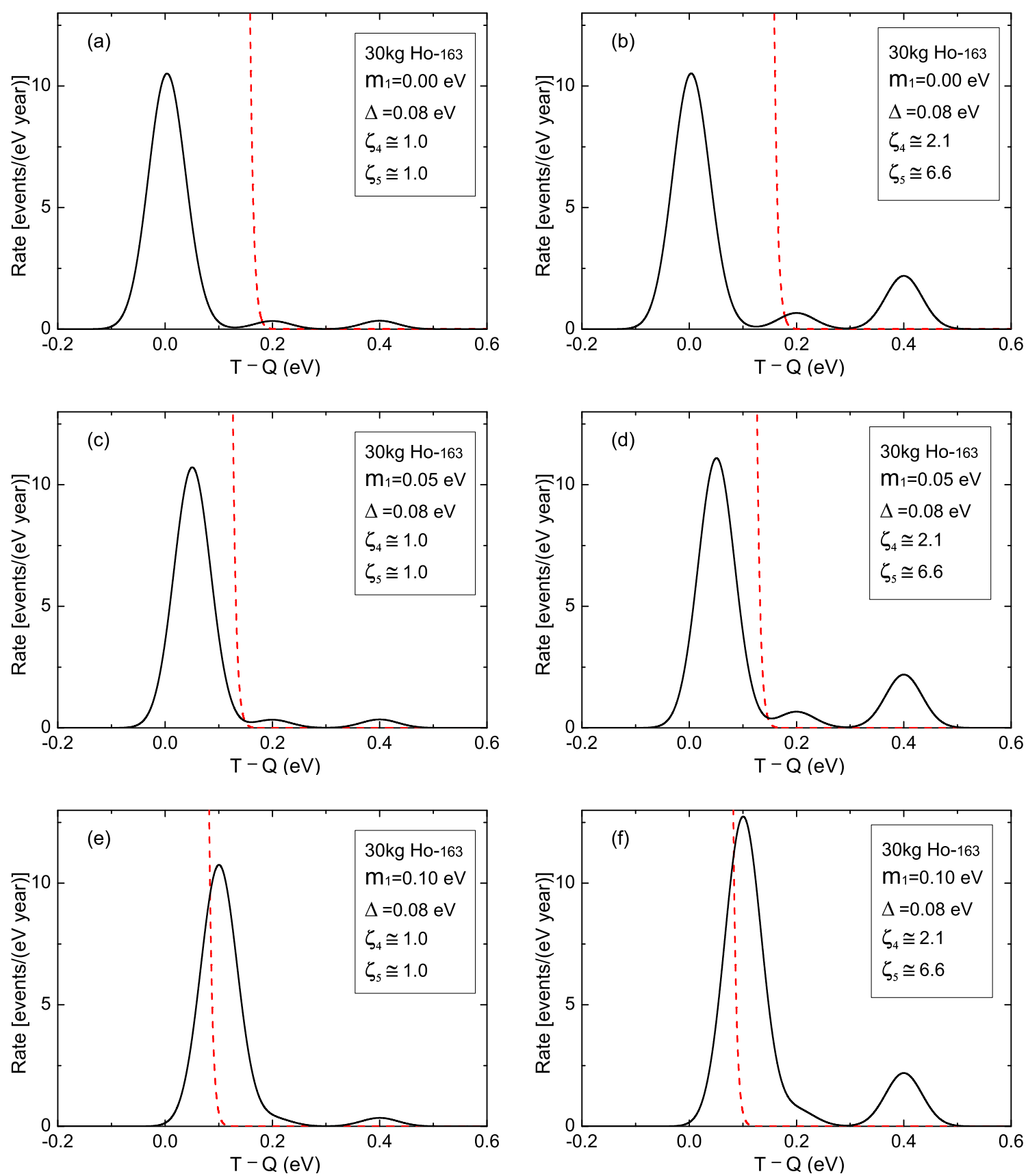

Figure 2: The relic antineutrino capture rate as a function of the overall energy release $T$ in the case of $\Delta m_{31}^{2}>0$, where $\zeta_{k}=1$ in the left panel and $\zeta_{k}>1$ described by Eq. (8) in the right panel to illustrate possible gravitational clustering effects on relic antineutrinos. The solid and dashed curves represent the $\mathrm{C} \bar{\nu} \mathrm{B}$ signature and its background, respectively. The value of the finite energy resolution $\Delta$ is taken in such a way that only the signal of the sterile component of the $\mathrm{C} \bar{\nu} \mathrm{B}$ can be seen. 

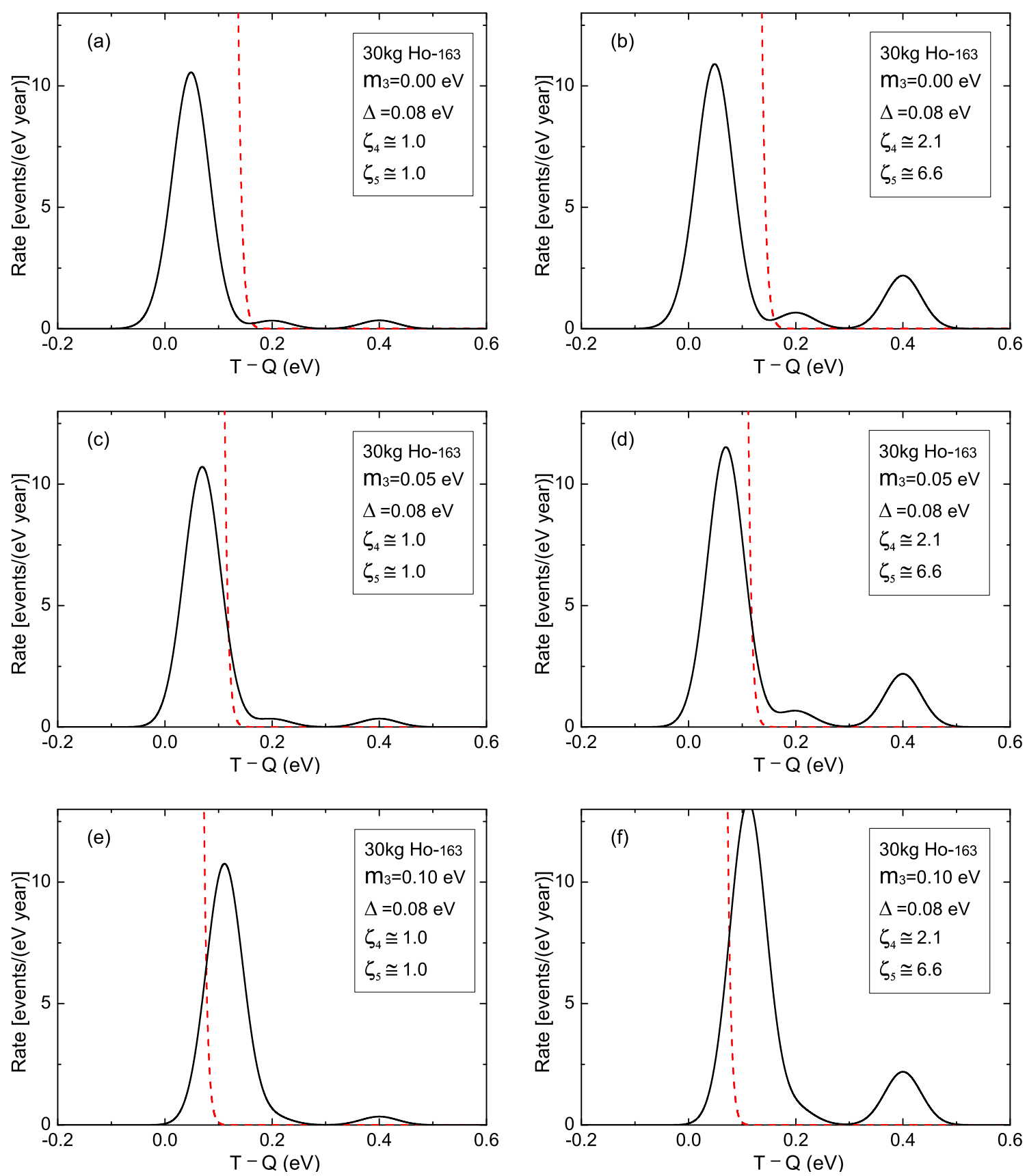

Figure 3: The relic antineutrino capture rate as a function of the overall energy release $T$ in the case of $\Delta m_{31}^{2}<0$, where $\zeta_{k}=1$ in the left panel and $\zeta_{k}>1$ described by Eq. (8) in the right panel to illustrate possible gravitational clustering effects on relic antineutrinos. The solid and dashed curves represent the $\mathrm{C} \bar{\nu} \mathrm{B}$ signature and its background, respectively. The value of the finite energy resolution $\Delta$ is taken in such a way that only the signal of the sterile component of the $\mathrm{C} \bar{\nu} \mathrm{B}$ can be seen. 


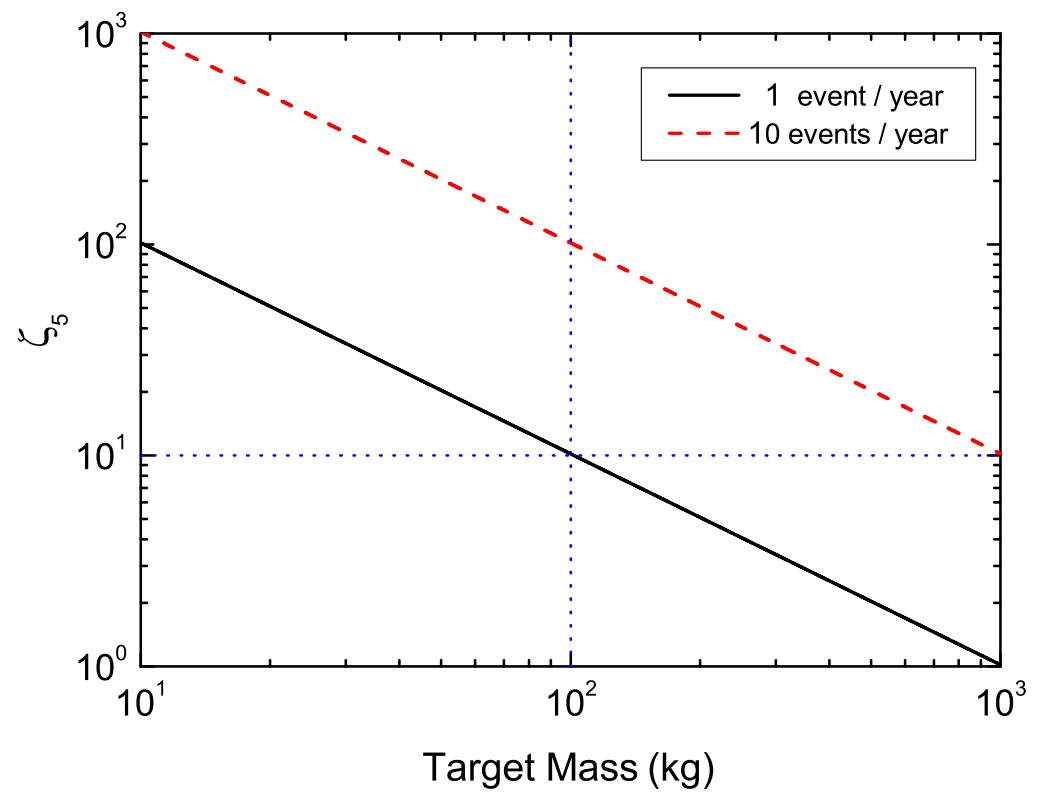

Figure 4: The iso-rate curves of hot sterile antineutrino DM for the overdensity parameter $\zeta_{5}$ versus the target mass. Here $\zeta_{5}$ is treated as a free parameter and $m_{5} \simeq 0.4 \mathrm{eV}$ and $\left|V_{e 5}\right| \simeq 0.174$ have been input.

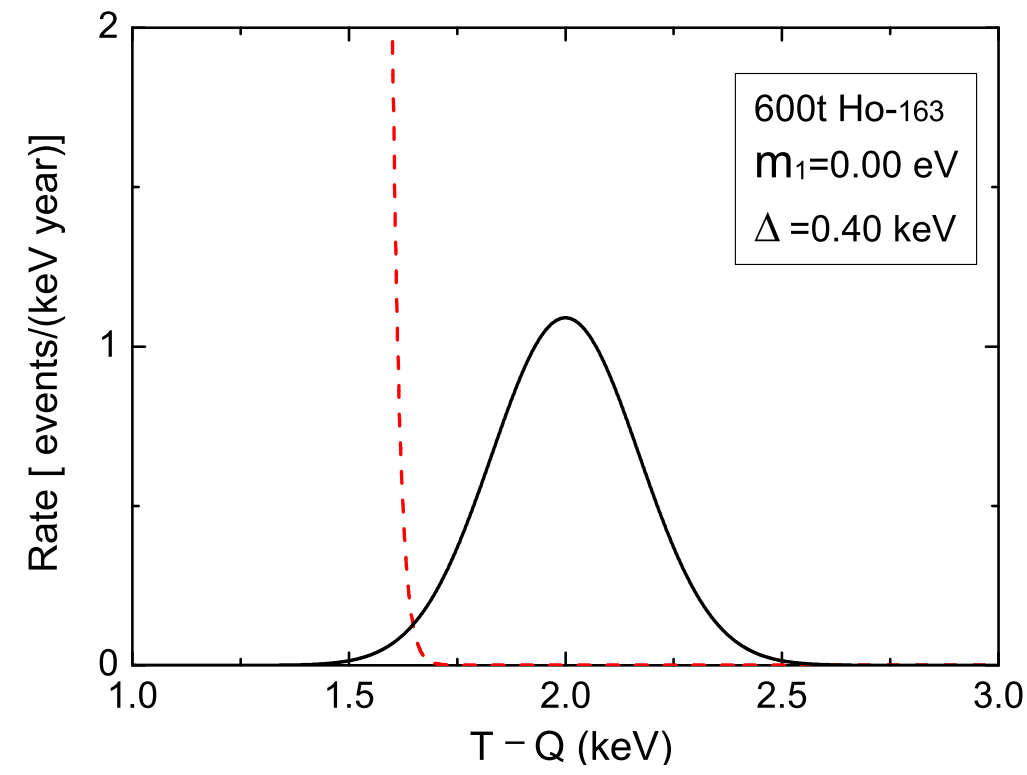

Figure 5: The rate of capturing warm sterile antineutrino DM as a function of the overall energy release $T$ in the (3+1) flavor mixing scheme with $\Delta m_{31}^{2}>0$ and $m_{4} \simeq 2 \mathrm{keV}$. The solid and dashed curves represent the signature and its EC-decay background, respectively. 


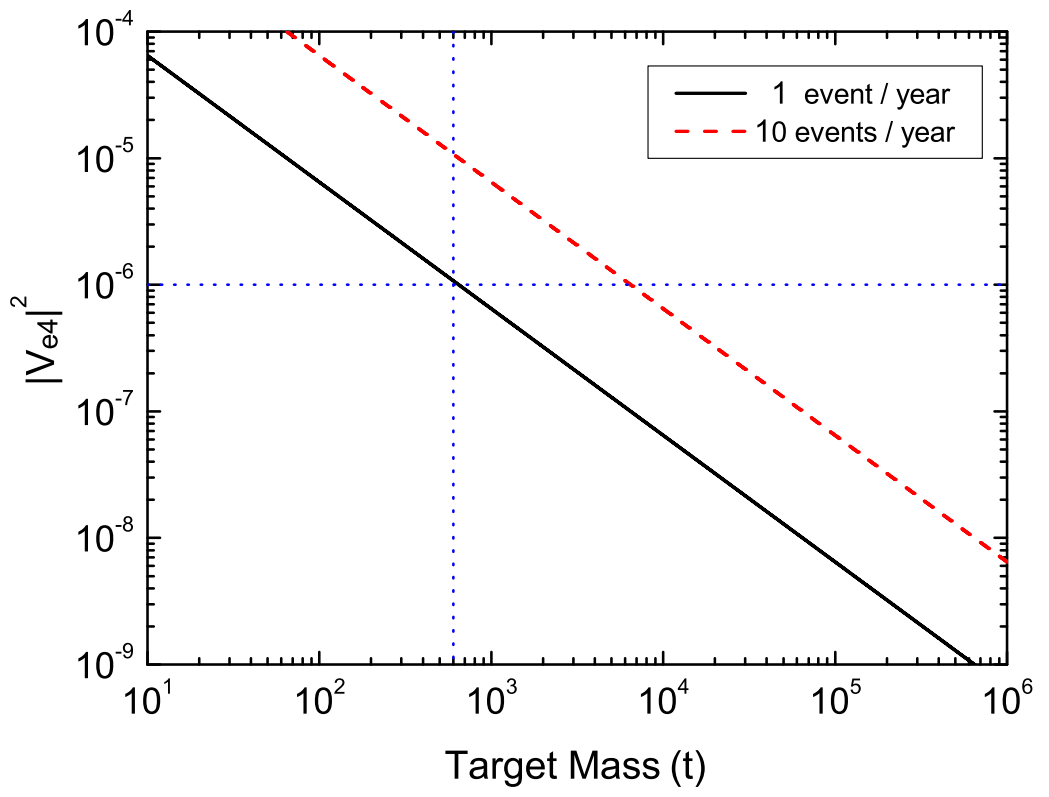

Figure 6: The iso-rate curves of warm sterile antineutrino DM for the mixing matrix element $\left|V_{e 4}\right|^{2}$ versus the target mass. Here $n_{\nu_{4}} \simeq n_{\bar{\nu}_{4}} \simeq 5 \times 10^{4}\left(3 \mathrm{keV} / m_{4}\right) \mathrm{cm}^{-3}$ in our Galactic neighborhood has been input. 